3. Verwendung zur Emulsionierung und Solidifizierung. Wasserlösliche Oele.

Herstellung von Sulfoverbindungen.

Auf dem Gebiete der Emulgierung von Mineralölen liegt nichts besonderes vor $\left.{ }^{1}\right)$, auch nicht auf dem der Solidifizierung (Verfestigung). Erwähnt seien die Patente von Lifschütz ${ }^{2}$ ) über wasserbindende Kohlenwasserstoffe.

$\mathrm{Ku}_{\mathrm{k}} \mathrm{ka}^{3}$ ) befasst sich eingehend mit den Liebreich'schen Patenten; ein Anonymus stellt die falschen Anschauungen, die über die -Verseifung * des Petroleums herrschen, richtig ").

1) D. P. $215140 \mathrm{~K}$ nopf (mit Hilfe von Seifen unter Zusatz uberschussiger Oelsäure und Alkohol); Krefelder Seifenfabrik, Fr. P. 396493 , Darstellung und Verwendung von Emulsionen; Boidin u. Soc. an. Amyls, Am. P. 906188, Löslichmachen von Kohlenwasserstofien; $\mathbf{v}$ an der Ploeg; Oe. P. 39337, Herstellung wässriger Emulsionen von schweren Kohlenwasserstoffen; D. P. 204061; Schröder, Verf. u. Vorr. $z$. stetigen Herstellung v, Emulsionen; Saporta, Fr. P. 400911, Schnelle Darstellung v. Emulsionen fluchtiger Oele durch einfaches Mischen mit Wasser; The Arabol Mfg. Co., Fr. 404 959, Emulgieren von Oelen und Fetten.

?) D. R. P. 205428; Oc. P. 39442; Behandlung der Kohlenwasserstoffe mit O-abgebenden Substanzen, wie Superoxyden anorganischer oder besser organischer Natur; ferner Wolf und Böhme, Belg. P. 213172: KohlenWasserstoffreiche Seifen.

) Chem. Rev. 1909, p. 30. p. 155

Seifens.-Ztg. 1908, p. 1165; Chem.-Ztg. Rep. 1909,
Mit den Petrolseifen befasst sich schliesslich auch ein Artikel in , Les matières grasses ${ }^{1}$ ).

Die Verwendung von Mineralölen zur Staubbindung hat im Hinblick auf die rapide Zunahme des Automobilismus steigendes In. teresse, ebenso die Verwendung von Fussbodenölen. Hierüber berichten How ard ${ }^{2}$ ), Wahrtre ${ }^{8}$ ), Bindewald") (über ,Kitons, Versuche mit. einem Gemisch Teer und Ton), Bernhard ${ }^{3}$.

Ueber eine neue, in Kalifornien ausgeführte Methode berichtet der britische Konsul in Los Angeles, die Kosten sollen die Häfte wie bisher betragen ${ }^{6}$ ).

1) Siehe J. du pétr. 1909 , p. 173, uber eine Analyse von iSimplicits (wasserlösliches Vaselinöl), siehe Chem. Rev. 1909, p. 232.

9) Die Verminderung des Strassenstaubes ... (Centralbl. f. Wasserbau 1908, 3, pp. 7/8; Chg. Rep. 1909, p. 135.

2) Chem. Techn. Ztg. 1909 , p. 133.

4) Techn. Gemeindebl. 1909, XII, Nr. 2, 3, 4; J. Gasb. 1909, 52, pp. 654/8; Chg. Rep. 1909 , p. 505.

) Untersuchungen uber die Ursachen der Bildung des Staubes .... und uber Versuche zur Bekämpfung desselben; Petr. 1909, IV, p. 694; uber wasserlösliche Oele zur Strassenbesprengung, siehe Seifens. Ztg. 1909, p. 714; uber staubbindende Fussbodenöle, die Abhandlung von $\mathrm{He}$ ise, Chem. Rev, 1909, p. 58; Chg. Rep. 1909, p. 246.

-) Oil. Col. Tr. 1909, p. 805, ferner Petr. Rev. 1909. XXI, p. 294; Einige Patente, Houben D. R. P. 206885; Engl. P. 16789, 1908: Staubbindendes Mittel; Ames: D. R. P. 205627 , Teeren von Strassen; Wer d: Am. P. 933803 , Teerstrassenpflaster.

(Schluss folgt.)

\title{
Ueber Jodzahlen des Linoxyns
}

(Mitteilung des Laboratoriums der chem. Fabrik Dr. F. Wilhelmi, Leipzig-R.) von Dipl.-Ing. Meister.

Bekanntlich nimmt die Jodzahl von Leinöl mit fortschreitender Oxydation des Oeles ständig ab. Die Sauerstoffaufnahme geht mit einer Abnahme des Jodaufnahmevermögens Hand in Hand. Schon längeres Lagern bei Luftzutritt erniedrigt die Jodzahl nicht unerheblich. Firnisse und gekochte Oele nehmen unter Umständen nur noch halb soviel Jod auf wie das ursprüngliche Leinöl. Ebenso bringt naturgemäss der Trockenprozess eine Erniedrigung der Jodzahl mit sich, der Theorie nach müsste dieselbe eigentlich bei alten Firnishäutchen auf Null herabsinken. Es stellen sich aber der Bestimmung der Jodzahl in den letzten Phasen des Trockenprozesses bedeutende Schwierigkeiten in den Weg, da sich das Linoxyn, je älter es wird, um so schwieriger in den anwendbaren Lösungsmitteln löst. Der einzige Weg, um schliesslich zu einer Jodzahl 0 zu kommen, der aber den Verhältnissén der Praxis in keiner Weise entspräche, wäre von vorn. herein mit Leinölfettsäure zu arbeiten, die ge- trockneten Firnishäutchen zu verseifen, mit Mineralsäure die Fettsäuren des Linoxyns abzuscheiden, wieder aufzustreichen und damit solange fortzufahren bis keine Erniedrigung der Jodzahl mehr eintritt oder das Jodaufnahmevermögen überhaupt aufhört. Das würde aber, wie gesagt, den Verhältnissen der Praxis nicht entsprechen und ausserdem auch kein klares Bild geben.

Ueber Jodzahlen oxydierter Leinöle und Linoxyne (Feste Leinöle ) ist besonders von Lew kowitsch gearbeitet worden. In seinem Werke Technologie und Analyse der Fette, Oele und Wachser hat er sich eingehend über diesen Gegenstand verbreitet und noch ausführlicher hat er hierüber in einer Abhandlung in dieser Zeitschrift (IX, p. 51) berichtet. Lewkowitsch gibt als Jodzahl für, festes Leinöl $\approx 52,2$ an. Er hat allerdings mit sogenanntem Scrim-Oel (in Deutschland meist WaltonOel genannt) gearbeitet und es ist erklärlich, dass ein derartiges Produkt, wie diese den 
Oxydierhäusern entstammende Linoleummasse kein reines, oxydiertes Leinöl, sondern ein $\mathrm{Ge}$ misch von oxydiertem und mehr oder weniger unverändertem Leinöl darstellt und mithin noch eine relativ hohe Jodzahl haben muss.

Leider ist mir nicht bekannt und in den betreffenden Literaturstellen auch nicht angegeben, welches Lösungsmittel Lewkowitsch bei diesen Jodzahlbestimmungen verwendet hat. Mit den üblichen Lösungsmitteln wie Chloroform, Tetrachlorkohlenstoff, Benzol usw. ist jedenfalls nicht viel anzufangen, auch mit ab. solutem Alkohol, mit welchem Lewkowitsch bei der Jodzahlbestimmung der Gesamtfettsäuren des Linoxyns gearbeitet hat, kommt man nicht vorwärts. Recht gut kann man aber das Linoxyn in Eisessig $(98 \%)$ lösen und in dieser Lösung die Jodzahl bestimmen. Aeltere Firnishaut lässt sich allerdings hierin kalt nicht völlig lösen und es bedarf einer gelinden Erwärmung um dieselbe in Lösung zu bringen. Je älter das Linoxyn ist, um so deutlicher kann man einen geringen, in Eisessig unlöslichen Rückstand erkennen. Also auch mit diesem Lösungsmittel kann man nicht ganz bis zum Ende kommen; wie unten angeführten Tabellen zu entnehmen ist, kann man den Trockenprozess auf diese Weise aber doch ziemlich weit ver. folgen, wenigstens bis zu dem Punkte, wo eine erhebliche Gewichtszunahme der Firnishaut nicht mehr konstatierbar ist.

Meines Erachtens bestehen keine Bedenken, Eisessig als Lösungsmittel zur Jodzahlbestimmung zu benutzen. Nach der Wijs'schen Methode wird ja auch mit einer Eisessig-Jodlösung gearbeitet. Jodzahlen derselben Leinölfettsäure, einmal in Chloroform, einmal in Eisessig gelöst, zeigten gute Uebereinstimmung. Möglich ist allerdings in den Fällen, wo sich eine warme Lösung des Linoxyns in Eisessig nötig macht, eine teilweise Azetylierung. $\mathrm{Da}$ aber bei einem Vorversuche heiss in Eisessig gelöste Leinölsäure dieselbe Jodzahl (innerhalb der zulässigen Fehlergrenze) ergab, wie dieselbe Fettsäure in Chloroform scheint das auf die Jodzahl keinen Einfluss $z u$ haben. Im Gegensatz zu der Linoleunmasse, muss in ausserordentlich dünner
Schicht bei einmaligem Aufstrich getrocknetes Leinöl ziemlich reines Linoxyn enthalten und demgemäss die mit diesem Produkte erhaltenen Jodzahlen niedriger liegen als die des Waltonöles.

Um nun die Jodzahlen in den verschiedenen Phasen des Trockenprozesses verfolgen und gleichzeitig eine möglichst vollständige $\mathrm{Oxy}$. dation erreichen zu können, wurde ein guter, in etwa sechs Stunden trocknender, mit $3 \%$ geschmolzenem Blei-Manganresinat bei $150^{\circ} \mathrm{C}$ hergestellter Leinölfirnis in dünner Schicht (etwa $0,5-0,7 \mathrm{mg}$ auf den $\square \mathrm{cm}$ ) auf mehreren grossen Glasplatten ausgebreitet. Zur Feststellung der Gewichtszunahme wurde derselbe Firnis gleichzeitig auf dünne, gewogene Glastäfelchen gestrichen und diese alle zwei Stunden gewogen. In den gleichen Zeiten wurde von den grossen Glastafeln Oel entnommen und die Jodzahl bestimmt. Die ersten beiden Male wurde in Chloroformlösung gearbeitet. Das dritte Mal (nach sechs Stunden) konnte das bereits festgewordene Oel nicht mehr in $\mathrm{CHCl}_{3}$ gelöst werden, liess sich aber leicht in kaltem Eisessig in Lösung bringen. Das vierte und fünfte Mal (nach 8 und 10 Stunden) musste mit warmem Eisessig gelöst werden. Die Jodzahl des fünften Versuchs ist in beiden Fällen nicht mehr als völlig genau anzusehen, weil sich gegen das Ende der Titration hin, allerdings verschwindend geringe, Mengen veränderten Linoxyns auf der Oberfläche abschieden.

Bei den Bestimmungen, bei welchen in warmem Eisessig gelöst wurde, war übrigens der Farbenumschlag nicht mehr ganz scharf, weil der letzte Tropfen Hyposulfitlösung die Jodlösung nicht ganz entfärbte, sondern dieselbe etwas grünstichig liess. Trotzdem ist der Farbenumschlag aus dem Blaugrün in's Helle noch recht gut zu erkennen.

Diese Versuche wurden mit zwei verschiedenen, etwa zwei Monate alten Leinölfirnissen an zwei verschiedenen Tagen ausgeführt. Im übrigen wurden die Jodzahlen nach der Hübl'schen Methode bestimmt und die Titrationen nach 18 stündigem Stehen vorgenommen.

Firnis I

\begin{tabular}{c|c|c|c|c|c|c|c}
\hline & Firnis & $\begin{array}{c}\text { nach } \\
\text { 2stüdigem } \\
\text { Trocknen }\end{array}$ & $\begin{array}{c}\text { nach } \\
\text { 4 stundigem } \\
\text { Trocknen }\end{array}$ & $\begin{array}{c}\text { nach } \\
\text { 6stundigem } \\
\text { Trocknen }\end{array}$ & $\begin{array}{c}\text { nach } \\
\text { 8stündigem } \\
\text { Trocknen }\end{array}$ & $\begin{array}{c}\text { nach } \\
\text { 10stundigem } \\
\text { Trocknen }\end{array}$ & $\begin{array}{c}\text { nach } \\
\text { 12 stündigem } \\
\text { Trocknen }\end{array}$ \\
\hline $\begin{array}{c}\text { Jodzahl...... } \\
\begin{array}{c}\text { Gewichtszu- } \\
\text { nahme in \% }\end{array}\end{array}$ & 153,8 & 131,6 & 105,0 & 73,9 & 47,3 & $26,9(3)$ & - \\
\end{tabular}


Firnis II

\begin{tabular}{|c|c|c|c|c|c|c|c|}
\hline & Firnis & $\begin{array}{c}\text { nach } \\
\text { 2 stidndigem } \\
\text { Trocknen }\end{array}$ & $\begin{array}{c}\text { nach } \\
\text { 4 stündigem } \\
\text { Trocknen }\end{array}$ & $\begin{array}{c}\text { nach } \\
\text { 6stündigem } \\
\text { Trocknen }\end{array}$ & $\begin{array}{c}\text { nach } \\
\text { 8stündigem } \\
\text { Trocknen }\end{array}$ & $\begin{array}{c}\text { nach } \\
\text { 10stindigem } \\
\text { Trocknen }\end{array}$ & $\begin{array}{c}\text { nach } \\
12 \text { stundigem } \\
\text { Trocknen }\end{array}$ \\
\hline Jodzahl & 156,8 & 136,1 & 104,0 & 74,8 & 48,8 & 29,1 (?) & - \\
\hline $\begin{array}{l}\text { Gewichtszu- } \\
\text { nahme in } \%\end{array}$ & - & 5,1 & 10,0 & 16,2 & 17,4 & 18,0 & 18,1 \\
\hline
\end{tabular}

Firnis I war nach sechs Stunden völlig trocken, Firnis II nach derselben Zeit noch ganz wenig klebrig.

Aller Wahrscheinlichkeit nach fällt die Jodzahl auch nach der zehnten Stunde noch weiter, da nach der zwölften Stunde eine, wenn auch geringe, Gewichtszunahme zu konstatieren war. Das Linoxyn war aber nach 12 Stunden Trockenzeit nicht mehr völlig in Lösung zu bringen. Der noch offene Weg, nach Verseifung und Abscheidung der Fettsäuren des Linoxyns die Jodzahl der betreffenden Gesamtfettsäuren zu bestimmen, wurde nicht beschritten, weil er nicht zu Resultaten führen konnte, welche sich den oben angeführten gleichmässig angliederten.

Das Linoxyn wird immer schwerer in Eisessig löslich; 48 Stunden nach dem Aufstrich lösten sich schätzungsweise nur noch $50 \%$.

Von drei Sorten Waltonöl, welche ich untersuchte, waren dagegen zwei, trotzdem sie schon vor längerer Zeit den Oxydierhäusern entnommen waren, ziemlich leicht in Eisessig löslich, die dritte bis auf einen ganz geringen Rückstand. Die beiden löslichen Sorten hatten Jodzahlen von 53,2 und 49,7 , kamen also den bei Lewkowitsch angegebenen Werten ziemlich nahe.

Ein Versuch, in gleicher Weise wie bei Leinölfirnis auch bei Holzölfirnis vorzugehen, führte nicht zu dem gewünschten Resultate, weil das nach vierstündigem Trocknen entstandene, matt aufgetrocknete Elaeostearin sich als unlöslich in Eisessig und allen anderen zu diesem $Z$ wecke herangezogenen Lösungsmitteln erwies.

Immerhin konnte entsprechend der geringen Gewichtszunahme nach zweistündigem Trocknen eine merkbare Abnahme der Jodzahl konstatiert werden.

Holzölfirnis I

\begin{tabular}{l|c|c|c}
\hline & Firnis & $\begin{array}{c}\text { nach } \\
\text { 2stündigem } \\
\text { Trocknen }\end{array}$ & $\begin{array}{c}\text { nach } \\
\text { 4stundigem } \\
\text { Trocknen }\end{array}$ \\
\hline $\begin{array}{l}\text { Jodzahl ... } \\
\begin{array}{l}\text { Gewichtszu- } \\
\text { nahme in } \%\end{array}\end{array}$ & 148,8 & 143,1 & - \\
\hline
\end{tabular}

Holzölfirnis II

\begin{tabular}{l|c|c|c}
\hline & Firnis & $\begin{array}{c}\text { nach } \\
\text { 2 stundigem } \\
\text { Trocknen }\end{array}$ & $\begin{array}{c}\text { nach } \\
\text { 4 stundigem } \\
\text { Trocknen }\end{array}$ \\
\hline $\begin{array}{l}\text { Jodzahl ... } \\
\begin{array}{l}\text { Gewichtszu- } \\
\text { nahme in } \%\end{array}\end{array}$ & 144,9 & 138,9 & - \\
\end{tabular}

Theoretisch dürfte eigentlich eine Gewichts. zunahme bei dem Uebergang des Elaeomargarins in das isomere Elaeostearin nicht stattfinden. $\mathrm{Ob}$ letzterem an und für sich eine niedere Jodzahl zukommt als dem Elaeomargarin, konnte durch diese Versuche natürlich nicht festgestellt werden.

\section{Zusammensetzung einiger 0ele und Trane. (Mitgeteilt von A. P. Lidoff in Charkow.)}

\section{Oel aus den Samen von Ulmus campestris.}

\section{Pavlenko.}

Aus den Samen wurden erhalten $26,09 \%$ Oel bei der Extraktion mit Benzin:

Spezifisches Gewicht bei $20^{\circ} \mathrm{C} \quad 0,9559$

Erstarrungspunkt ........ $3,5^{\circ} \mathrm{C}$

Schmelzpunkt ..........
Säurezahl . . . . . . . . . 5,57

Verseifungszahl ........ . 277,3

Hehner's Zahl (im Mittel von drei Bestimmungen) . . . . 75,45\%

Reichert's Zahl ........ 3,75

Jodzahl . . . . . . . . . . . 32,2

Gesamtstickstoffgehalt im extrahierten Mehle........4, 4, $16 \%$ 